\title{
Nucleosynthesis in thermonuclear supernovae
}

Ivo Seitenzahl and Dean Townsley

\begin{abstract}
The explosion energy of thermonuclear (Type Ia) supernovae is derived from the difference in nuclear binding energy liberated in the explosive fusion of light "fuel" nuclei, predominantly carbon and oxygen, into more tightly bound nuclear "ash" dominated by iron and silicon group elements. The very same explosive thermonuclear fusion event is also one of the major processes contributing to the nucleosynthesis of the heavy elements, in particular the iron-group elements. For example, most of the iron and manganese in the Sun and its planetary system was produced in thermonuclear supernovae. Here, we review the physics of explosive thermonuclear burning in carbon-oxygen white dwarf material and the methodologies utilized in calculating predicted nucleosynthesis from hydrodynamic explosion models. While the dominant explosion scenario remains unclear, many aspects of the nuclear combustion and nucleosynthesis are common to all models and must occur in some form in order to produce the observed yields. We summarize the predicted nucleosynthetic yields for existing explosions models, placing particular emphasis on characteristic differences in the nucleosynthetic signatures of the different suggested scenarios leading to Type Ia supernovae. Following this, we discuss how these signatures compare with observations of several individual supernovae, remnants, and the composition of material in our Galaxy and galaxy clusters.
\end{abstract}

Ivo Rolf Seitenzahl

Research School of Astronomy and Astrophysics, Australian National University, Canberra, ACT 2611, Australia e-mail: ivoseitenzahlegmail.com

Dean Martin Townsley

Department of Physics and Astronomy, The University of Alabama, Tuscaloosa, AL 35487-0324

e-mail: dean.m.townsley@ua.edu 


\section{Introduction}

"Thermonuclear supernovae" is the collective name given to the family of supernova explosions that derive the energy that drives their expansion against gravity from the exothermic transmutation of less tightly bound nuclear "fuel" (such as e.g. ${ }^{4} \mathrm{He},{ }^{12} \mathrm{C}$, or ${ }^{16} \mathrm{O}$ ) into more tightly bound nuclear "ashes" (such as e.g. ${ }^{28} \mathrm{Si}$ or ${ }^{56} \mathrm{Ni}$ ). This is in contrast to "core-collapse supernovae", which, in spite of a contribution to the explosion energy by nuclear burning behind the outgoing shock-wave, are largely powered by the gravitational binding energy that is released during the stellar core collapse. A key difference between the thermonuclear burning in thermonuclear supernovae and core-collapse supernovae and is that the nucleosynthesis in the former generally proceeds at higher fuel density and therefore at lower entropy. For a (text) book on supernovae and nucleosynthesis see Arnett (1996).

Although details of the progenitor system and explosion mechanism are still unknown, there is a general consensus that thermonuclear supernovae are the physical mechanism behind what are observationally classified as "Type Ia supernovae" (SNe Ia); the two terms are therefore often used interchangeably. Note that while it is likely that all SNe Ia are of thermonuclear origin, the converse need not be true. A key aspect all sub-classes of thermonuclear supernova models have in common is that they all feature explosive (meaning dynamical) thermonuclear burning in white dwarfs (WD), stars that are supported against gravitation collapse by electron degeneracy pressure.

We begin by summarizing the physical processes of explosive fusion involved in thermonuclear supernovae and how the interplay of these with the structure and dynamics of the exploding WD lead to the major yields of the explosion. A short discussion of the techniques used to compute yields in modern simulations follows. Our discussion of the expectations for the yields of thermonuclear supernovae then proceeds by treating several of the major proposed scenarios for the explosion. The nucleosynthesis of p-nuclei are discussed separately, and we end the chapter with a discussion of the observational signatures of various aspects of nucleosynthesis in thermonuclear supernovae.

\section{Physics background and methodology}

\subsection{Explosive thermonuclear burning}

The key fundamental aspect of nucleosynthesis in a Type Ia supernova comes from the realization, established by Nomoto et al (1984), that if a near-Chandrasekhar mass carbon-oxygen (CO) WD star is incinerated on a similar timescale to its dynamical time, the resulting ejecta gives rise to a transient that is spectroscopically characteristic of a Type Ia. That is, it is poor in $\mathrm{H}$ and $\mathrm{He}$ and shows strong $\mathrm{Si}$ features at maximum light, with a power source interior to the maximum light pho- 
tosphere provided by the radioactive decay of ${ }^{56} \mathrm{Ni}$. Exactly how such an incineration of a WD comes about, and even where the massive CO WD required by some scenarios might come from are still mysterious, but the basic model remains clear.

Some time spent on why incineration of a WD leads to the observed synthesized ejecta is worthwhile. The density structure of a hydrostatic WD, along with the density dependence of the nucleosynthetic outcome of explosive $\mathrm{CO}$ fusion, lead naturally to an ejecta structure in which iron group elements (IGE), including ${ }^{56} \mathrm{Ni}$, make up the inner regions, surrounded by $\mathrm{Si}$-rich outer layers. Figure 1 shows roughly how the products of burning depend on the local fuel density, with high densities leading to more complete processing to IGE in nuclear statistical equilibrium (NSE), and lower densities truncating the nuclear processing with the production of Si-group intermediate mass elements (IME). In NSE, material is hot and dense enough that all nuclear reactions that preserve the number of protons and neutrons can be approximated as occuring quickly. As a result, the composition is determined by the relative binding energies of various species and statistical mechanical considerations and does not depend on individual reaction rates or, in a computation, their uncertainty.

Also shown in Figure 1 is the density structure of a near-Chandrasekhar mass $\left(M_{\mathrm{Ch}}\right) \mathrm{WD}\left(1.38 \mathrm{M}_{\odot}\right)$, as well as a $1.05 \mathrm{M}_{\odot} \mathrm{WD}$. It is immediately clear that the hydrostatic state of the WD at the time the burning takes place has a direct role in the nucleosynthetic yield produced. A $M_{C h}$ WD burned by a detonation, for example, with no opportunity to expand, will produce nearly all IGE. Comparatively, a $1.05 \mathrm{M}_{\odot}$ star subjected to a detonation will produce only about $0.6 M_{\odot}$ of IGE, with the balance being mostly IME, all simply due to the lower overall density. A similar

Fig. 1 Nucleosynthetic products are determined principally by the density of the fuel when the reaction front passes. High densities lead to more complete burning. Profiles of WDs of several masses are shown, demonstrating that a WD close to the Chandrasekhar mass (solid green), if burned without any expansion, produces almost solely IGE, while a $1.05 \mathrm{M}_{\odot}$ WD (dashed orange), will not produce Mn or stable IGE due to electron capture. In the delayed-detonation family of scenarios, early burning expands the $M_{\mathrm{Ch}}$ WD to a state in which significant amounts of IME are produced upon full incineration (dot-dashed blue).

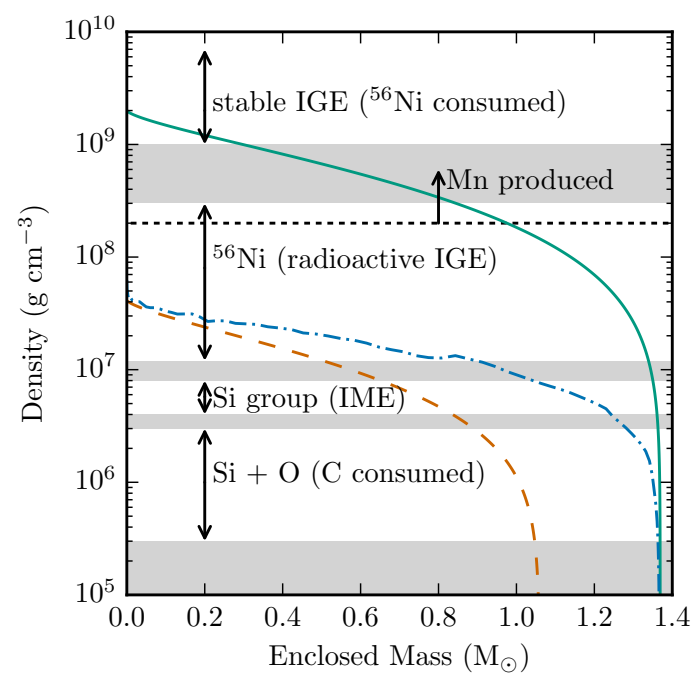


balance of yields favoring more IME can be obtained if the beginning of the incineration occurs slightly more slowly than the dynamical time of the star (around 1 second). Then the star can expand as shown in the middle curve in Figure 11, so that the lowered densities lead to the production of IME in the outer region.

We will only discuss the physics of the relevant modes of combustion briefly. The deflagration, or flame, mode is more slowly propagating and therefore able to allow the star to globally respond and expand. Its propagation is driven by heat conduction in the degenerate electron medium. The faster mode, detonation, is propagated by a shock that is self-sustained by the energy release and propagates across the star in less than the dynamical time.

The observed nucleosynthesis of SNe Ia shows evidence of a large fraction of stable IGE in the innermost regions of the ejecta (e.g., Mazzali et al, 2007, 2015). As seen in Figure 1, synthesis of stable IGE requires densities above a few $10^{8} \mathrm{~g} \mathrm{~cm}^{-3}$. These densities are only present in CO WDs above about $1.2 \mathrm{M}_{\odot}$, which can only be formed by accretion. Conversion of protons to neutrons by capture of electrons proceeds at high densities because the Fermi energy of the degenerate electrons, which hold the star up against gravity, is high enough for this conversion to be favorable. At densities above a few $10^{8} \mathrm{~g} \mathrm{~cm}^{-3}$ the conversion rate is fast enough that, in less than the dynamical time of the expanding star, the ${ }^{56} \mathrm{Ni}$-dominated NSE is changed first into one in which ${ }^{54} \mathrm{Fe}$ and ${ }^{58} \mathrm{Ni}$, both of which are stable, are the dominant species. Longer exposure can lead to even more neutron-rich products. Comparison of the balance of neutron-enriched and non-enriched isotopes produced by the explosion to those found in the solar distribution constrains the central density during the initial part of the explosion (Brachwitz et al, 2000). These observational constraints are important due to uncertainty in the ignition physics (e.g., Gasques et al, 2005, 2007).

In addition to its importance for the isotopic abundances, neutron enrichment also decreases the amount of radioactive ${ }^{56} \mathrm{Ni}$ available to power the observed bright transient. The overall neutron richness of the material is determined by both the amount of electron capture during the supernova, as just described, as well the initial metallicity and electron capture during the pre-explosion core convection phase. The initial metallicity controls the initial neutron excess mostly via the abundance of ${ }^{22} \mathrm{Ne}$ in the progenitor WD (Timmes et al, 2003). Almost all the initial C, N, and $\mathrm{O}$ elements in the progenitor star are first converted to ${ }^{14} \mathrm{~N}$ in the H-burning CNO cycle. Then during the He burning phase that forms the CO WD, $\alpha$ captures and beta decay convert this ${ }^{14} \mathrm{~N}$ into ${ }^{22} \mathrm{Ne}$. Core ignition of a thermonuclear $\mathrm{SN}$ is preceded by a phase of convective core carbon burning. During this phase, electron capture near the center increases neutron enrichment (Chamulak et al, 2008, Piro and Bildsten, 2008: Martínez-Rodríguez et al, 2016), with significant uncertainty due to the convective Urca process (Stein and Wheeler, 2006). For sub- $M_{\mathrm{Ch}}$ scenarios, the settling of the ${ }^{22} \mathrm{Ne}$ within the WD (Bildsten and Hall 2001) may be important for the distribution of stable IGE in the ejecta. Metallicity can also change the explosion in other ways (Calder et al, 2013) including modification of the DDT density (Jackson et al, 2010). 
Another product only obtained in large quantities from high-density burning is manganese (Seitenzahl et al, 2013a). This is produced as ${ }^{55} \mathrm{Co}$ in the explosion and then decays. Figure 1 shows the dividing line, at about $2 \times 10^{8} \mathrm{~g} \mathrm{~cm}^{-3}$, between alpha-rich freeze-out of NSE at lower densities and normal NSE freeze-out at higher densities. At high densities, and therefore comparatively lower entropies for similar energy deposited, as the temperature falls, freeze-out occurs as the reactions that maintain the NSE become starved of $\alpha$ and other light particles. By comparison, somewhat lower densities produce entropies at which $\alpha$ and other light particles are still present during freeze-out and destroy some species present in the NSE, including ${ }^{55} \mathrm{Co}$. The solar ratio of $\mathrm{Mn}$ to $\mathrm{Fe}$ is higher than that produced by core-collapse $\mathrm{SNe}$, thus requiring production in thermonuclear $\mathrm{SNe}$. This therefore implies a significant fraction of events with material burned above this threshold density (Seitenzahl et al 2013a), another indication of near- $M_{\mathrm{Ch}}$ progenitors.

\subsection{Methodology}

Two aspects of the combustion length scales in SNe Ia make it challenging to compute accurate yields in explosion simulations. Both of these are related to the relatively small length and time scales on which the reactions involved occur (For a summary see the introduction of Townsley et al, 2016). While the WD is of order several $10^{8} \mathrm{~cm}$ across, the length scales of the combustion front can be microns for the highest densities to $\mathrm{cm}$ for moderate densities $\sim 10^{7} \mathrm{~g} \mathrm{~cm}^{-3}$. In addition to this, the contrast in reaction length and time scales within the reaction front can be similarly vast. At $10^{7} \mathrm{~g} \mathrm{~cm}^{-3}$, the length scale for full conversion to IGE in a planar detonation is $\sim 10^{8} \mathrm{~cm}$, while the peak $\mathrm{Si}$ abundance only occurs some $10^{3} \mathrm{~cm}$ behind the shock, and the carbon consumption scale is of order $\mathrm{cm}$ (Seitenzahl et al, 2009a, Townsley et al, 2016). These two contrasts in length scale make it computationally infeasible to compute the reaction front structure explicitly in simulations of the explosion of the star.

Another challenge comes from the number of species involved in the nuclear reactions. Accurate computation of carbon combustion with a nuclear reaction network (Hix and Meyer, 2006) requires of order 200-300 species in order to accurately capture combustion, silicon burning, and finally the electron capture at high densities (Calder et al, 2007; Seitenzahl et al, 2009c). Larger reaction networks can give a few percent improvement in accuracy, but around 200-300 appears to be sufficient. For most of the history of SNe Ia simulations, it was not possible to perform star-scale fluid dynamics simulations with such a large number of species. This is currently improving with current very large machines having a high degree of parallelism in each node, but there will continue to be a trade-off between fidelity of nuclear processes with more species and resolution of hydrodynamic processes such as turbulence with more spatial cells.

These two challenges, of unresolved reaction length scales and the preference for good spatial resolution in multi-dimensional simulations, preferably 3D, instead 
of many species, have led to the current strategy in which the reactions in the explosion simulation are modeled in some way and then the yields are determined via post-processing of Lagrangian tracers (Travaglio et al, 2004, Seitenzahl et al, 2010; Townsley et al, 2016). The post-processing proceeds by using Lagrangian fluid histories, often called "tracks," "trajectories," or "tracers," recorded at many places in the hydrodynamic simulation of the explosion. Two examples of the density and temperature history tracks recorded in this manner from a 2-dimensional simulation (assuming azimuthal symmetry) of the deflagration-detonation transition (DDT) scenario (Townsley et al, 2016) are shown in Figure 2. The top two panels show tracks for fluid elements that were processed in the detonation (solid) and deflagration (dashed) modes. Another advantage of separating the hydrodynamics and nucleosynthesis is that the influence of some changes, such as minor rates or initial abundances, can be evaluated based on a smaller number of hydrodynamic simulations (e.g. Bravo and Martínez-Pinedo, 2012, Parikh et al, 2013; Miles et al, 2016).

This strategy of computing the explosion using large eddy simulation (LES) is neither unique to astrophysics nor to SNe Ia. In other contexts it may be used in circumstances where the physical turbulence dissipation scale is unresolved, but the behavior of turbulence in small scales is relatively well understood from the Kol-

Fig. 2 Nucleosynthesis is computed by processing the temperature-density history of a fluid element, shown in the top two panels, computed in a hydrodynamic simulation of the explosion. The lower two panels show the resulting abundance neutron excess and abundance history. The solid lines show a track processed by the detonation which only partially processes IME to IGE. Only a few major abundances are shown: ${ }^{12} \mathrm{C}$ (red), ${ }^{28} \mathrm{Si}$ (green), and ${ }^{56} \mathrm{Ni}$ (black). The dashed lines show a track processed by the deflagration which has the ${ }^{56} \mathrm{Ni}$ yield reduced by electron capture, enhancing the neutron excess $\eta_{n}$. The short blue sections in the upper panels are the portion of the time history that is reconstructed (Townsley et al 2016.

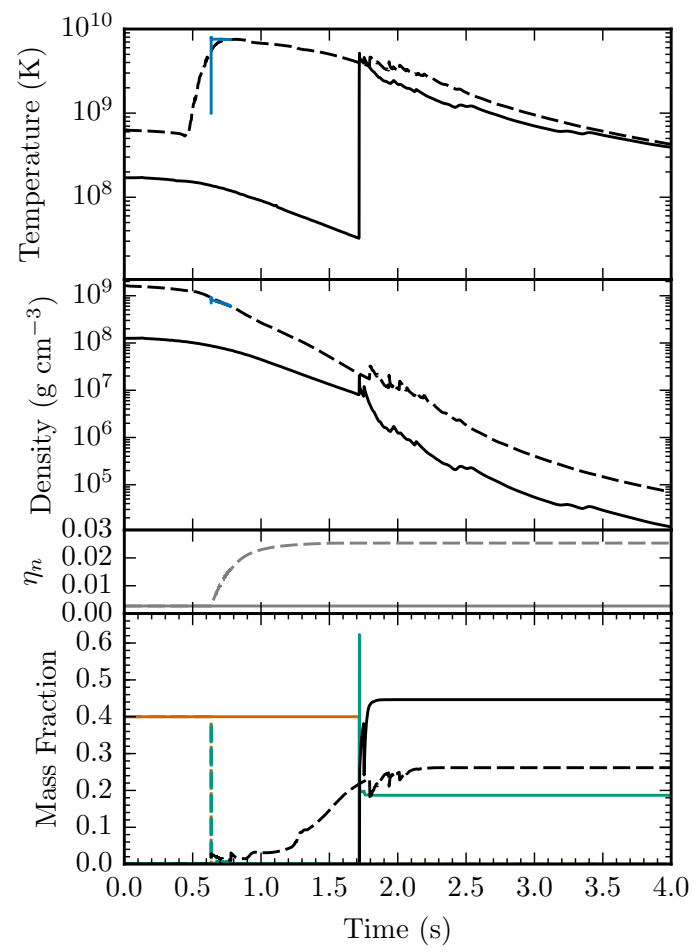


mogorov model of turbulence and its successors. As a result, it is possible to do a valid simulation by only explicitly simulating the largest scales in an LES. Supernovae present the additional complication of reactions, which are also subgrid scale like the turbulent dissipation. The necessity of having a combustion model that accurately captures unresolved phenomena then introduces the topic of calibration and verification of that model. The development of improved combustion models for LES and their verification is therefore a central topic in simulation of $\mathrm{SNe}$ Ia and continues as increasingly detailed comparisons to observations are performed (Schmidt et al, 2006, Seitenzahl et al, 2010; Ciaraldi-Schoolmann et al, 2013; Jackson et al, 2014; Townsley et al, 2016). In addition to uncertainty due to the modelling of unresolved processes in the hydrodynamics, there are also reaction rate uncertainties (Bravo and Martínez-Pinedo, 2012, Parikh et al, 2013).

Examples of two tracks processed under different conditions in the same explosion, taken from Townsley et al (2016), are shown in Figure 2 The solid lines show the track for a fluid parcel that undergoes combustion via a detonation late in the simulation, about $1.7 \mathrm{~s}$. As can be seen, most of the nuclear processing of this parcel occurs near the reaction front or within about $0.2 \mathrm{~s}$ after the detonation passage. The density when the detonation shock arrives is around $10^{7} \mathrm{~g} \mathrm{~cm}^{-3}$, leading to incomplete burning of $\mathrm{Si}$, as seen in the final abundances for this track, which have a mixture of IME and IGE. No significant electron capture takes place so that the neutron excess $\eta_{n}=\sum_{i}\left(N_{i}-Z_{i}\right) Y_{i}=\sum_{i}\left(N_{i}+Z_{i}\right) Y_{i}-2 \sum_{i} Z_{i} Y_{i}=1-2 Y_{e}$ is unchanged. Here $Y_{e}$ is the number of electrons per baryon, $Y_{i}$ is the number of nuclei of species $i$ per baryon, $N_{i}$ and $Z_{i}$ are the number of neutrons and protons in the nucleus of species $i$, and charge balance is assumed. The dashed lines show the track for a fluid parcel processed by the deflagration earlier in the simulation, about $0.6 \mathrm{~s}$. The deflagration track's final abundances are markedly different from the detonation, mostly because the chosen track shows a significant degree of conversion of protons to neutrons by electron capture, as show by the difference in $\eta_{n}$. Immediately after passage of the deflagration front, all the fuel as well as the synthesized Si have been destroyed, and the ${ }^{56} \mathrm{Ni}$ fraction is low due the high-temperature, high-density NSE state; much of the material is in the form of $\alpha$ particles. As the material cools and expands, the ${ }^{56} \mathrm{Ni}$ abundance rises, but only to a fraction of about 0.3 by mass, because most of the IGE are in the form of more neutron rich species like ${ }^{54} \mathrm{Fe}$ and ${ }^{58} \mathrm{Ni}$. While both detonation and deflagration fronts are unresolved in this $4 \mathrm{~km}$ resolution simulation, in Figure 2 it is much more obvious for the deflagration case because the reaction front moves more slowly. At the deflagration propagation speed, which is $\sim \mathrm{km} \mathrm{s}^{-1}$, thousands of times less than the sound speed, it takes several tenths of a second for a fluid parcel to pass through the thickened reaction front that is a few times the grid resolution in thickness. In order to improve yield accuracy in the context of this artificial broadening, Townsley et al (2016) developed a technique in which part of the thermal history recorded during the simulation is replaced in post-processing by a separate computation of the fully resolved structure of a steady-state deflagration (blue curve in upper panels). The temperature rise in such a reconstruction is much faster, giving a more physically realistic temperature peak and therefore total integrated amount of electron captures. 


\section{Explosive nucleosynthesis predictions of thermonuclear supernova models}

We give here only a broad overview that focuses on the qualitative differences of existing models in their respective nucleosynthetic yields. There are many different ways of categorizing thermonuclear SNe. From a nucleosynthesis point of view, it makes most sense to group the explosion models into $i$ ) near- $M_{c h}$ models (involving deflagrations ignited at high density and the associated neutronization) and $i i$ ) pure detonation models of less massive WDs and iii) models including detonations of thick He-shells and the special nucleosynthesis products thereof.

\subsection{Thermonuclear SNe from near- $M_{C h}$ primary WDs}

These models have in common the near central ignition of a deflagration via pycnonuclear fusion reactions as the accreting primary WD grows in mass and the central density increases. From a nucleosynthesis standpoint, these are generally the only models where in situ electron captures significantly lower the electron fraction and drive the yields towards more neutron-rich isotopes. Further, these are the only models where low entropy "normal" freeze-out from NSE occurs, with its typical nucleosynthetic signature, such as enhanced production of Mn (see discussion in Section 5). For these two reasons, deflagrations in high central density $\left(\rho \gtrsim 5 \times 10^{9} \mathrm{~g} \mathrm{~cm}^{-3}\right.$ ) WDs have been suggested as the only plausible production site of certain neutron-rich intermediate mass and iron-group isotopes, such as ${ }^{48} \mathrm{Ca}$, ${ }^{50} \mathrm{Ti}$, or ${ }^{54} \mathrm{Cr}$ (see discussion in Section 5). Since in-situ electron captures significantly increase the neutron-excess after ignition, the ignition density of the WD can have a greater effect on the final yields (e.g., Seitenzahl et al, 2011) than the metallicity of the progenitor star (e.g., Miles et al, 2016).

\subsubsection{Pure (turbulent) deflagrations}

A detonation in a hydrostatic near- $M_{c h}$ CO WD (Arnett, 1969) would burn most of the star at high density to ${ }^{56} \mathrm{Ni}$ and other IGE, producing insufficient IMEs such as $\mathrm{Si}$ and $\mathrm{S}$ to explain observed spectra of SNe Ia. This has lead to deflagrations as the long-time favoured model, since a sub-sonic flame allows the WD to respond to the nuclear energy release with expansion to lower densities where the flame can produce IMEs. The widely referenced $\mathrm{W} 7$ model of a deflagration in a $1.38 \mathrm{M}_{\odot} \mathrm{CO}$ WD (Nomoto et al, 1984) has been very successful in reproducing key elements of the inferred structure of normal SNe Ia, such as the mass of ${ }^{56} \mathrm{Ni}$ and the layered profile of the IMEs. However, W7 overproduced neutron rich nuclear species such as e.g., ${ }^{54} \mathrm{Cr}$, ${ }^{54} \mathrm{Fe}$, or ${ }^{58} \mathrm{Ni}$ (Thielemann et al, 1986, Iwamoto et al, 1999), a shortcoming that was ameliorated (Brachwitz et al, 2000) when the electron capture rates 
of Fuller et al (1982) were replaced with new electron capture rates for pf-shell nuclei from Langanke and Martínez-Pinedo (2001). The initially slow evolution of the flame and the suppression of buoyancy still leads to enhanced electron captures in the W7 model even for the updated electron capture rates and hence too much ${ }^{58} \mathrm{Ni}$ (Maeda et al, 2010). The one-dimensional W7 model implements a sub-sonically moving flame that moves outward in the mass-coordinate, which means buoyancy and turbulent mixing are suppressed. As an unphysical consequence, the flame overruns the whole star and the burning ashes do not mix, retaining their original positions in the mass-coordinate. Three-dimensional pure deflagration models, which take buoyancy, fluid instabilities, and mixing into account, are too faint and chemically mixed to explain normal SNe Ia (e.g., Röpke et al, 2007) and their colors and spectra do not agree (Fink et al, 2014). Weakly ignited pure deflagration models that only partially unbind the star and leave a bound remnant behind do, however, provide excellent models for sub-luminous SNe Iax (Kromer et al, 2013, 2015). While the ejecta of deflagration models that fail to completely unbind the whole WD (Fink et al, 2014) are rich in IGE and contain little unburned ${ }^{12} \mathrm{C}$ and ${ }^{16} \mathrm{O}$, more strongly ignited deflagrations that unbind the whole WD, such as e.g. the N1600 model from Fink et al (2014), eject $>0.3 \mathrm{M}_{\odot}$ of both ${ }^{12} \mathrm{C}$ and ${ }^{16} \mathrm{O}$, in addition to large amounts $\left(0.1 \mathrm{M}_{\odot}\right.$ of ${ }^{54} \mathrm{Fe}$ and $0.07 \mathrm{M}_{\odot}$ of $\left.{ }^{58} \mathrm{Ni}\right)$ of neutron-rich stable iron group isotopes, as well as $[\mathrm{Mn} / \mathrm{Fe}]>0$. Here we use the widespread "bracket" notation: the logarithm of the ratio of element A to element B relative to the corresponding ratio in the Sun, $[A / B]=\log _{10}(A / B)-\log _{10}(A / B) \odot$.

\subsubsection{Deflagration to detonation transition (DDT) models}

To overcome the nucleosynthetic shortcomings of pure detonation and pure deflagration models, Khokhlov (1991) introduced a delayed-detonation model, where an initial the sub-sonic deflagration can transition to a super-sonic detonation under suitable conditions. The expansion of the star prior to the detonation results in the desired nuclear burning at densities $\lesssim 10^{7} \mathrm{~g} \mathrm{~cm}^{-3}$ where IMEs such as Si and S are synthesized. The significant contribution of the deflagration to IGE nucleosynthesis however still leaves an imprint on the overall yields, with overall $[\mathrm{Mn} / \mathrm{Fe}]>0$ and (slightly) super-solar ${ }^{54} \mathrm{Fe} /{ }^{56} \mathrm{Fe}$ and ${ }^{58} \mathrm{Ni} /{ }^{56} \mathrm{Fe}$ (Seitenzahl et al, 2013b). The white dwarf's carbon fraction (or C/O ratio) is only a secondary parameter; it does not influence the nucleosythesis in $M_{c h}$ models a lot since a lot of material burns into NSE (Ohlmann et al 2014). DDT models tend to produce low light $\alpha$-element to Fe ratios, with typical $\mathrm{O}$ yields around $0.1 \mathrm{M}_{\odot}$ and $\mathrm{Ne}$ and $\mathrm{Mg}$ yields around $0.01 \mathrm{M}_{\odot}$ or lower.

\subsubsection{GCD and PRD models}

The so-called gravitationally confined detonation (GCD) model (Plewa et al, 2004) is based on a deflagration that ignites off-center in a single bubble and a detonation 
that is triggered just under the WD surface on the opposite side of the ignition point, when the deflagration ash compresses unburned fuel there after rising towards the surface and expanding laterally around the star. For this type of explosion model, detailed nucleosynthesis calculations that go beyond a basic 13 isotope $\alpha$-network have been presented for 2D simulations by Meakin et al (2009) and for 3D simulations by Seitenzahl et al (2016). A key signature of GCD models is that although it is technically a near- $M_{c h}$ explosion model, very little mass is burned in the deflagration. As such, their isotopic nucleosynthesis signature resembles more that of pure detonations in massive sub-Chandrasekhar mass WDs, such as presented in Marquardt et al (2015). [Mn/Fe] is sub-solar and products of neutronization, in particular the stable iron-group isotopes ${ }^{54} \mathrm{Fe}$ and ${ }^{58} \mathrm{Ni}$, are not not overly abundant. Burning is quite complete, only $\sim 0.1 \mathrm{M}_{\odot}{ }^{16} \mathrm{O}$ and a few $\times 10^{-2} \mathrm{M}_{\odot}$ of ${ }^{12} \mathrm{C}$ survive the explosion.

The pulsating reverse detonation (PRD) model (Bravo and García-Senz, 2009) evolves initially analogous to the GCD model from a weak deflagration, although here the detonation is though to be triggered when an accretion shock forms during the contraction phase of the WD after the first radial pulsation brought about by the energy released in the deflagration. Since again not very much mass, $0.14-0.26$ $\mathrm{M}_{\odot}$ in the Bravo et al (2009) models, is burned in the deflagration, the nucleosynthesis products do not carry the typical signature of near- $M_{c h}$ models, for example, $[\mathrm{Mn} / \mathrm{Fe}]$ is sub-solar (Bravo et al, 2009).

\subsection{Thermonuclear SNe from detonating sub-M $M_{C h} W D s$}

Next, we group and discuss explosion models that are fundamentally based on detonations in sub- $M_{c h}$ WDs. Compared to near- $M_{c h}$ models, the smaller mass of the detonating primaries means that explosive nuclear burning at densities above $\sim 10^{8} \mathrm{~g} \mathrm{~cm}^{-3}$ does generally not contribute to the yields. Consequently, the typical nucleosynthetic signatures of deflagrations, such as $[\mathrm{Mn} / \mathrm{Fe}]>0$ or copious production of electron capture nuclei ${ }^{58} \mathrm{Ni}$ or ${ }^{54} \mathrm{Fe}$, are collectively absent in this group of models. In the following, we briefly discuss the main proposed scenarios of detonating sub- $M_{c h}$ WDs, once again with a focus on their nucleosynthetic signatures.

\subsubsection{Violent WD+WD merger}

For a mass ratio close to unity, the violent merger of two WDs is thought to provide a means to detonate at least one of the two WDs to produce a thermonuclear SN. The mass of the primary (heavier) WD is hereby the most important factor in determining whether the SN is sub-luminous (Pakmor et al, 2010), of "normal" SN Ia luminosity (Pakmor et al 2012), or over-luminous (Moll et al. 2014), since in the violent merger models the primary WD detonates close to hydrostatic equilibrium (cf. Sim et al, 2010, Ruiter et al, 2013). A small layer of helium present on the primary is 
thought to be essential in achieving robust detonations (Pakmor et al, 2013). Generally, the densities achieved in violent mergers are too low for low entropy freeze-out from NSE or enhanced production of neutron-rich electron capture nuclei (e.g., ${ }^{54} \mathrm{Fe}$ or ${ }^{58} \mathrm{Ni}$ ) to occur. The isotopic composition of the IGEs therefore directly reflects the neutron excess and hence the progenitor system metallicity mainly through the abundance of ${ }^{22} \mathrm{Ne}$ (see section 2).

Whether or not the secondary WD detonates or gets disrupted is particularly important for setting the the overall production ratios, especially affecting the nucleosynthesis of the intermediate mass $\alpha$-elements, with a detonation in the lower mass secondary mostly (depending on the exact mass) ejecting ${ }^{12} \mathrm{C},{ }^{16} \mathrm{O},{ }^{28} \mathrm{Si}$, and other IMEs (Pakmor et al, 2012).

\subsubsection{Double-detonation models with He-shells}

WDs accreting $\mathrm{H}$ at a low accretion rate $\lesssim$ few $\times 10^{-8} \mathrm{M}_{\odot} \mathrm{yr}^{-1}$ process the $\mathrm{H}$ to $\mathrm{He}$ and accumulate thick He-shells that may ignite explosively (e.g., Taam, 1980). Similarly, such thick He-shells can be accumulated by directly accreting He, either from a degenerate He WD (Tutukov and Yungelson, 1996) or a non-degenerate He-star (Iben et al, 1987). Multi-dimensional simulations have shown that detonations in the He-layer compress the core and likely (at least for high-mass $\mathrm{CO}$ cores) initiate a second CO detonation there (e.g., Livne, 1990, Woosley and Weaver, 1994, Shen and Bildsten, 2014), providing a possible explosion scenario for SNe Ia.

Detonations in thick helium-shells of $\gtrsim 0.1 \mathrm{M}_{\odot}$ process enough material in the He-detonation to have a global impact on the yields; in particular $\alpha$-isotopes such as ${ }^{36} \mathrm{Ar},{ }^{40} \mathrm{Ca},{ }^{44} \mathrm{Ti}$ (decaying to stable ${ }^{44} \mathrm{Ca}$ ), ${ }^{48} \mathrm{Cr}$ (decaying to stable ${ }^{48} \mathrm{Ti}$ ), or ${ }^{52} \mathrm{Fe}$ (decaying to stable ${ }^{52} \mathrm{Cr}$ ) are significantly enhanced compared to the products of explosive CO-burning (e.g., Woosley and Weaver, 1994, Livne and Arnett, 1995). The mass fraction of metals (e.g, C, N, O, Ne, ...) in the He-layer has a significant influence on the final nucleosynthesis products (e.g., Kromer et al, 2010; Waldman et al, 2011; Moore et al, 2013, Shen and Moore, 2014) and complicates model predictions.

Double-detonation explosion models with relatively large primary WD masses $\gtrsim 0.9 \mathrm{M}_{\odot}$ and relatively small He-shell masses $<0.1 \mathrm{M}_{\odot}$ provide an overall good match to observed "normal" SNe Ia, although current models still show some disagreement in the spectral and color evolution (e.g., Kromer et al, 2010, Fink et al, 2010; Woosley and Kasen. 2011). Systems with lower mass primary WDs have been proposed by Waldman et al (2011) as potential progenitor systems of Ca-rich Gap transients (Kasliwal et al, 2012) (SN 2005E-like events). Waldman et al (2011) present detailed nucleosynthesis calculations for a range of low mass CO WD + He shell models. 


\subsubsection{Detonating ONe WDs}

Detonations may also be possible for scenarios where the primary star is an $\mathrm{ONe}$ WD (Marquardt et al, 2015). Compared to a generic CO primary WDs (e.g., Sim et al, 2010), the greater mass and hence compactness of the ONe WD results in an ejecta strongly dominated by Fe-group isotopes, with about $0.1 \mathrm{M}_{\odot}$ of ${ }^{28} \mathrm{Si}$ and $\lesssim 0.05 \mathrm{M}_{\odot}$ of ${ }^{16} \mathrm{O}$ ejected by the primary explosion (Marquardt et al 2015 ). Naturally, very little carbon and direct products of carbon burning, such as $\mathrm{Na}$ or $\mathrm{Ne}$, are ejected by detonating ONe WDs.

\subsection{WD+WD collisions}

Papish and Perets (2016) present detailed isotopic yields for a range of CO WD + CO WD collision models, both with and without He-shells of various thickness. From a nucleosynthesis point of view, the important message is that the compression achieved during the collision (for the models investigated) is not enough to reach densities required for the copious production of electron capture nuclei or low-entropy freeze-out from NSE. The considerations for the nucleosynthesis of detonations in sub- $M_{c h}$ WDs and in He-shells therefore apply.

\section{4 p-nuclei nucleosynthesis}

In the previous sections we have reviewed how explosive thermonuclear fusion of light nuclei such as ${ }^{4} \mathrm{He},{ }^{12} \mathrm{C}$, and ${ }^{16} \mathrm{O}$ can synthesize heavier nuclei up to the iron-peak. The majority of the even heavier nuclides is synthesized by neutron capture processes, specifically the s- and r-processes (Cameron, 1957, Burbidge et al, 1957). A number of stable nuclides on the proton-rich side of the nuclear valleyof-stability however are "excluded" (Cameron, 1957) and cannot be synthesized by neutron capture processes as they are either bypassed or shielded from the s- and $\mathrm{r}$ process paths by more neutron-rich stable isobars. The main p-nuclei are ${ }^{74} \mathrm{Se},{ }^{78} \mathrm{Kr}$, ${ }^{84} \mathrm{Sr},{ }^{92,94} \mathrm{Mo},{ }^{96,98} \mathrm{Ru},{ }^{102} \mathrm{Pd},{ }^{106,108} \mathrm{Cd},{ }^{113} \mathrm{In},{ }^{112,114} \mathrm{Sn},{ }^{120} \mathrm{Te},{ }^{124,126} \mathrm{Xe},{ }^{130,132} \mathrm{Ba}$, ${ }^{138} \mathrm{La},{ }^{136,138} \mathrm{Ce},{ }^{144} \mathrm{Sm},{ }^{156,158} \mathrm{Dy},{ }^{162} \mathrm{Er},{ }^{168} \mathrm{Yb},{ }^{174} \mathrm{Hf},{ }^{180} \mathrm{Ta},{ }^{180} \mathrm{~W},{ }^{184} \mathrm{Os},{ }^{190} \mathrm{Pt}$, and ${ }^{196} \mathrm{Hg}$. p-nuclei contain less than one percent of the mass of nuclei heavier than $A=74$ in the Sun (Anders and Grevesse, 1989), yet they challenge our understanding of the synthesis of the heavy elements. The p-nuclei are thought to be synthesized via photo-disintegration $(\gamma, \mathrm{n})$-reactions of pre-existing s-process nuclei (e.g., Audouze and Truran 1975) in the secondary $\gamma$-process (Woosley and Howard, 1978), requiring temperatures in the range $2.0 \mathrm{GK} \lesssim T \lesssim 3.5 \mathrm{GK}$; for reviews on pnuclei and their nucleosynthesis see Lambert (1992); Pignatari et al (2016). The nucleosynthesis site of the p-nuclei has been under debate for 60 years. Core-collapse supernovae (CCSNe) have long been favored (Arnould, 1976), but even modern 
calculations fail to reproduce the solar abundance pattern, most notably still underproducing the light p-nuclei $A \lesssim 124$ (Rauscher et al, 2013). The origin of the ${ }^{92,94} \mathrm{Mo}$ and ${ }^{96,98} \mathrm{Ru}$ isotopes, which together comprise an unusually large $24 \%$ and $7 \%$ of the naturally occurring elements Mo and Ru respectively, has been particularly challenging to explain with CCSNe, due to a shortage of the relevant s-process seed nuclei (Woosley and Howard, 1978).

A further proposed site for p-nuclei nucleosynthesis are SNe Ia (Howard et al, 1991). Scenarios where the progenitor system is in the single degenerate scenario are particularly promising, since the s-process seed abundance can be enhanced significantly in the thermal helium pulses during the accretion phase, with ${ }^{12} \mathrm{C}(p, \gamma){ }^{13} \mathrm{~N}\left(e^{+} v_{e}\right){ }^{13} \mathrm{C}(\alpha, n){ }^{16} \mathrm{O}$ as the main neutron source (Iben, 1981). This in situ enhancement facilitates a high p-nuclei yield in general agreement with solar abundances when the s-process seed is processed by the SN Ia explosion (Travaglio et al, 2011). Whether CCSNe or SNe Ia were the dominant sites of the nucleosynthesis of the p-nuclei in the Sun is still unsettled. However, SNe Ia are currently the only viable proposed solution to the persistent ${ }^{92,94} \mathrm{Mo}$ and ${ }^{96,98} \mathrm{Ru}$ problem (Travaglio et al, 2011). Post-processing 2D delayed-detonation explosion model and performing Galactic chemical evolution calculations, (Travaglio et al, 2015) find that more than $50 \%$ of the p-nuclei (including Mo and Ru isotopes) can be synthesized by $\mathrm{SNe}$ Ia, making SNe Ia potentially the dominant site of the p-nuclei.

\section{Direct and indirect observable signatures of nucleosynthesis}

In this final section we discuss the observable signatures of the nucleosynthesis occurring in thermonuclear supernovae. First, we summarize the current state of different approaches aimed at inferring isotopic (as opposed to elemental) production masses synthesized in the explosions. The most prominent isotope is ${ }^{56} \mathrm{Ni}$, whose decay chain ${ }^{56} \mathrm{Ni} \stackrel{6 d}{\longrightarrow}{ }^{56} \mathrm{Co} \stackrel{77 d}{\longrightarrow}{ }^{56} \mathrm{Fe}$ supplies most of the heat (in the form of interacting $\gamma$-rays and positrons) that make the ejecta of SNe Ia shine brightly in optical light during the first $\sim 3$ years after the explosion.

The mass of ${ }^{56} \mathrm{Ni}$ synthesized in SNe Ia can be approximately determined by Arnett's rule: In its essence, Arnett's rule states that the bolometric luminosity of a SN Ia at maximum light is proportional (with proportionality constant close to unity) to the instantaneous energy deposition rate by the radioactive decay (Arnett. 1982), which is dominated by the ${ }^{56} \mathrm{Ni}$ decay chain at that time. Thus, the ${ }^{56} \mathrm{Ni}$ mass of a SN Ia can be directly estimated from the peak luminosity in a linear way. Although Arnett's rule is only approximate and contains some dependency on the explosion energy, opacity, total ejecta mass, or the spatial distribution of the ${ }^{56} \mathrm{Ni}$ (Pinto and Eastman 2000), it has been shown to yield ${ }^{56} \mathrm{Ni}$ masses that are consistent with those derived from more sophisticated radiative transfer modeling of the nebular spectra of SNe Ia (Stritzinger et al 2006). While Arnett's rule applied to normal SNe Ia yields typical ${ }^{56} \mathrm{Ni}$ masses in the relatively narrow range $0.3 \mathrm{M}_{\odot} \lesssim$ $M\left({ }^{56} \mathrm{Ni}\right) \lesssim 0.8 \mathrm{M}_{\odot}($ Scalzo et al, 2014$)$, the range covered by putative thermonuclear 
$\mathrm{SN}$ events including all sub-classes is much larger, spanning from significantly more than $1.4 \mathrm{M}_{\odot}$, e.g., SN 2007 if (Scalzo et al 2010), to just a few times $10^{-3} \mathrm{M}_{\odot}$, e.g., SN 2008ha (Foley et al, 2009).

For a handful of events it has been possible to more directly determine isotopic production masses of a few radionuclides by observing the characteristic $\gamma$-and $\mathrm{X}$-rays emitted in their decays. We now discuss these special events briefly in turn.

SN 2014J: A mere $3.5 \mathrm{Mpc}$ away, SN 2014J in M82 has been the closest Type Ia supernova since SN 1972E. Due to its proximity, the SPI gamma-ray spectrometer on the INTEGRAL satellite was able to directly detect for the first time the $\gamma$-ray lines from the decay of ${ }^{56} \mathrm{Ni}$ (Diehl et al, 2014) and ${ }^{56} \mathrm{Co}$ (Churazov et al, 2014) synthesized in a SN Ia. The total mass of ${ }^{56} \mathrm{Ni}$ derived from the $\gamma$-ray observations is around $0.6 \mathrm{M}_{\odot}$ (e.g., Churazov et al, 2015), in good agreement with estimates from Arnett's rule (Dhawan et al, 2016) and unambiguously confirming that the ${ }^{56} \mathrm{Ni}$ decay chain indeed powers the early light curves of normal SNe Ia.

G1.9+0.3: G1.9+0.3 is the youngest known supernova remnant in our Galaxy, with a putative explosion date around 1900 (Carlton et al, 2011). From detections of the $4.1 \mathrm{keV}$ X-ray line of Sc with the Chandra satellite, Borkowski et al (2010) infer a production mass of $1-7 \times 10^{-5} \mathrm{M}_{\odot}$ of ${ }^{44} \mathrm{Ti}$, in good agreement with the theoretical predictions for the leading explosion models, but ruling out models with detonations of thick He-shells for this particular event.

Tycho's SNR: In 2014 Troja et al (2014) reported an excess in their observations of Tycho's SNR in the $60-85 \mathrm{keV}$ band of the Burst Array Telescope (BAT) on the Swift satellite. However, their derived $3 \sigma$ range for the flux in the $78 \mathrm{keV}$ line, $2.4 \times 10^{-6}<F_{78}<2.6 \times 10^{5}$ photons $\mathrm{cm}^{-2} \mathrm{~s}^{-1}$ is not very restrictive, especially given the rather uncertain distance to Tycho's SNR. For a distance of $2.5 \mathrm{kpc}$, their $3 \sigma$ range corresponds to $3 \times 10^{-5} \lesssim M\left({ }^{44} \mathrm{Ti}\right) \lesssim 3 \times 10^{-4}$, which is in agreement with most of the explosion models discussion in section 3, but again ruling out the double-detonation model. In $\sim 750 \mathrm{ks}$ of NuSTAR observations Lopez et al (2015) do not detect any sign of ${ }^{44} \mathrm{Ti}$ and they note that in order for their non-detection to be consistent with the Swift/BAT results, the Ti cannot be at low velocity in the inner $2^{\prime}$. To be compatible, they require moderate or high-velocity ${ }^{44} \mathrm{Ti}\left(v \gtrsim 7,000 \mathrm{~km} \mathrm{~s}^{-1}\right)$ and/or that the ${ }^{44} \mathrm{Ti}$ is distributed across most of the extend of the SNR $\left(\gtrsim 3^{\prime}\right)$.

In addition to inferring isotopic production masses from direct line detections, there are indirect methods that can be used to infer isotopic yields. Although much less ${ }^{57} \mathrm{Ni}$ than ${ }^{56} \mathrm{Ni}$ is synthesized in the explosion and ${ }^{57} \mathrm{Co}$ does not emit any positrons, Seitenzahl et al (2009b) pointed out that, due to the longer half-life of ${ }^{57} \mathrm{Co}$ compared to ${ }^{56} \mathrm{Co}$, the $A=57$ decay chain ${ }^{57} \mathrm{Ni} \stackrel{36 h}{\longrightarrow}{ }^{57} \mathrm{Co} \stackrel{272 d}{\longrightarrow}{ }^{57} \mathrm{Fe}$ would none-the-less become the dominant radioactive energy source, heating the ejecta with the kinetic energy of internal conversion and Auger electrons emitted in the decay of ${ }^{57} \mathrm{Co}$. At even later times, ${ }^{55} \mathrm{Fe}$ (produced as ${ }^{55} \mathrm{Co}$ ) will become the dominant source of radioactive heating. Different explosion models predict different production ratios between the main radionuclides ${ }^{56} \mathrm{Ni},{ }^{57} \mathrm{Ni},{ }^{55} \mathrm{Co}$, and ${ }^{44} \mathrm{Ti}$. Knowledge of these production ratios, determined for example from modelling late-time observations of nearby $\mathrm{SNe}$ Ia, can therefore be used to discriminate between competing explosion models (Röpke et al, 2012), even for comparable ${ }^{56} \mathrm{Ni}$ masses. For two 
nearby SNe Ia it has been possible to indirectly infer a production mass in the $A=57$ decay chain from the interaction of the decay radiation with the ejecta.

SN 2011fe, a low velocity gradient (cf. Benetti et al, 2005) normal SN Ia that exploded a mere $6.4 \mathrm{Mpc}$ away in a relatively un-obscured region in M101, has been followed to very late times (e.g., Kerzendorf et al, 2014). Modeling the nebular spectrum from the epoch of 1034 days presented in Taubenberger et al (2015), Fransson and Jerkstrand (2015) conclude that ${ }^{57} \mathrm{Co}$ is required to explain the observed flux level at $\sim 1000 \mathrm{~d}$. Although they do not derive a best-fit production value for the mass of ${ }^{57} \mathrm{Ni}$, they show that the assumed 1.5 times solar production ratio of ${ }^{57} \mathrm{Ni}$ to ${ }^{56} \mathrm{Ni}$ under-predicts the observed flux by a factor of $\sim 2$, which indicates that the $57 / 56$ ratio of SN $2011 \mathrm{fe}$ was likely even greater.

SN 2012cg, also a normal SN Ia, was a bit further ( $\sim 15.2 \mathrm{Mpc}$ in NGC 4424) away than SN 2011fe, but HST photometry was still possible out to 1055 days past maximum light. $\mathrm{A}^{57} \mathrm{Ni}$ to ${ }^{56} \mathrm{Ni}$ production ratio of roughly twice the corresponding solar value provides the best fit to the observed light curve (Graur et al, 2016). After SN 2011fe, this is the second normal SN Ia that seems to require a ${ }^{57} \mathrm{Ni}$ to ${ }^{56} \mathrm{Ni}$ production ratio of $\gtrsim 2$ times solar. Since explosion models that involve exploding WDs near- $M_{c h}$ (such as delayed-detonations) predict greater 57/56 ratios than competing explosion models with significantly less massive primary WDs (such as the violent merger models or double-detonation models), Graur et al (2016) conclude that a near- $M_{c h}$ progenitor is more likely for SN $2012 \mathrm{cg}$.

As a major nucleosythesis site of heavy elements, thermonuclear supernovae also have a significant impact on Galactic chemical evolution (GCE); for a review on GCE see McWilliam (1997). Notably, SNe Ia are thought to have produced approximately two thirds of the Fe present in our Galaxy today (Dwek, 2016), the other third coming from CCSNe. CCSNe originate from massive stars and explode rather promptly after star-formation, whereas SNe Ia also arise in old stellar populations, which results in a "delayed" enrichment of the interstellar medium by SNe Ia. Any differences between CCSNe and SNe Ia in their nucleosynthetic yields ratios therefore result in gradients of those elemental abundance ratios as a function of metal enrichment, or $[\mathrm{Fe} / \mathrm{H}]$. For example, compared to CCSNe, the production ratios of the light $\alpha$-elements (in particular $\mathrm{O}, \mathrm{Ne}, \mathrm{Mg}$ ) relative to $\mathrm{Fe}$ are much lower in $\mathrm{SNe}$ Ia, which explains the observed decrease of $[\alpha / \mathrm{Fe}]$ with increasing $[\mathrm{Fe} / \mathrm{H}]$ for $[\mathrm{Fe} / \mathrm{H}]>-1$ (e.g., Timmes et al, 1995, Kobayashi and Nomoto, 2009). In addition to $\mathrm{Fe}$, SNe Ia are also the dominant production sites of $\mathrm{Cr}$ and $\mathrm{Mn}$, as well as contributing significantly, perhaps even the majority, to other iron group elements (such as $\mathrm{Ti}, \mathrm{Ni}$ ) and the heavier $\alpha$-elements, such as $\mathrm{Si}, \mathrm{S}, \mathrm{Ar}, \mathrm{Ca}$ (e.g., de Plaa, 2013).

Of all these, the mono-isotopic $\mathrm{Mn}$ is of particular interest. $[\mathrm{Mn} / \mathrm{Fe}]$ indicates that $\mathrm{SNe}$ Ia from near- $M_{c h}$ WD primaries cannot be uncommon, since they are the only viable proposed nucleosynthesis site that predicts $[\mathrm{Mn} / \mathrm{Fe}]>0$. The reason for this is that high density is required for the entropy to be low enough such that ${ }^{55} \mathrm{Co}$, which decays to ${ }^{55} \mathrm{Mn}$ via ${ }^{55} \mathrm{Fe}$, largely survives the freeze-out from NSE. This provides strong evidence that explosive nuclear burning at high density, $\rho \gtrsim 2 \times 10^{8} \mathrm{~g} \mathrm{~cm}^{-3}$, must have contributed significantly to the synthesis of $\mathrm{Fe}$ in the Galaxy (Seitenzahl et al, 2013a). It is worth noting here that this strongly den- 
sity/entropy dependent ${ }^{55} \mathrm{Co}$ yield can be used as a model discriminant for individual, nearby SNe via the $5.9 \mathrm{keV} \mathrm{X-ray} \mathrm{emission} \mathrm{from} \mathrm{the} \mathrm{decay} \mathrm{of}{ }^{55} \mathrm{Fe}$ (Seitenzahl et al 2015) or via the effect of radioactive heating by X-rays and Auger electrons from decaying ${ }^{55} \mathrm{Fe}$ (Röpke et al 2012). Of interest in the context of GCE are further the chemical peculiarities of Local Group dwarf galaxies (Kobayashi et al, 2015), which could be explained by the nucleosynthetic contribution of SNe Iax, in particular pure deflagration models that fail to completely unbind the WD and leave remnants behind (e.g., Kromer et al, 2013, 2015).

X-ray spectroscopy of hot intra-cluster medium (ICM) has emerged as one of the most promising ways to measure chemical abundances to constrain SN explosion models (e.g., Dupke and White, 2000, de Plaa et al, 2007). The hot ICM is generally optically thin to X-rays and close to collisional ionization equilibrium (see e.g., Dopita and Sutherland, 2003), making abundance measurements relatively straightforward (for a review see e.g., Böhringer and Werner 2010). The gravitational potential of large galaxy clusters is deep enough to retain the SN ejecta and the metal abundance of the ICM therefore directly reflects the integrated yields of all $\mathrm{SNe}$ (core collapse and thermonuclear) up to the present. Fits to the observed ICM abundances (Mernier et al, 2016a) indicate that to explain the large abundances of $\mathrm{Ar}$ and $\mathrm{Ca}$ (and perhaps Cr) (de Plaa, 2013, Mernier et al, 2016b), a further component has to be invoked, in addition to the contributions from normal SNe Ia and CCSNe. Since no such contribution is required to explain the abundances in the Sun (Mernier et al, 2016b), this points at very old stellar populations (long delay-time) giving rise to these explosions. A promising match to these requirements could be 1991 bg-like $\mathrm{SNe}$, a scenario that could simultaneously explain the origin and morphology of the Galactic $511 \mathrm{keV}$ anti-matter annihilation line (Crocker et al .2016). Moreover, once again based on the Mn abundance, it is disfavored that most SNe Ia originate from detonating sub- $M_{c h}$ WDs (Mernier et al, 2016b).

X-ray observations of supernova remnants can provide further independent information about the chemical elements synthesized by SNe Ia and constrain explosion scenarios for individual SNRs. For example, the high $\mathrm{Mn} / \mathrm{Fe}$ and $\mathrm{Ni} / \mathrm{Fe}$ ratios determined for SNR 3C 397 from Suzaku observations is indicative of neutronized material that could only be explained by an exploding massive near- $M_{c h} \mathrm{WD}$ (Yamaguchi et al, 2015).

Last but not least, the nucleosynthesis origin of a few neutron-rich intermediate mass and iron group isotopes is very likely also linked to thermonuclear $\mathrm{SNe}$, in particular for ${ }^{48} \mathrm{Ca}$, but also ${ }^{50} \mathrm{Ti},{ }^{54} \mathrm{Cr}$, and others (e.g., Woosley, 1997). Meyer et al (1996) showed that nucleosynthesis of ${ }^{48} \mathrm{Ca}$ cannot primarily occur in CCSNe since the formation and subsequent survival of the ${ }^{48} \mathrm{Ca}$ quasi-equilibrium cluster (Bodansky et al, 1968; Woosley et al, 1973) not only requires high neutron excess but also low entropy, such as is only obtained in explosive thermonuclear burning at the highest densities obtained in near- $M_{c h}$ SN Ia explosions, for example from explosive thermonuclear burning in ONeMg WDs. Recent three-dimensional simulations by Jones et al (2016) cast serious doubt on the canonical wisdom that near- $M_{c h}$ accreting ONeMg WDs collapse to neutron stars after central $\mathrm{Ne}$ and O-burning is 
ignited, opening the exciting possibility that such events may indeed be the primary nucleosynthesis site of ${ }^{48} \mathrm{Ca}$ and a few other neutron rich isotopes.

Acknowledgements IRS was supported during this work by Australian Research Council Laureate Grant FL09921.

\section{References}

Anders E, Grevesse N (1989) Abundances of the elements - Meteoritic and solar. Geochimica et Cosmochimica Acta53:197-214, DOI 10.1016/0016-7037(89)90286-X

Arnett D (1996) Supernovae and Nucleosynthesis: An Investigation of the History of Matter from the Big Bang to the Present, Princeton University Press, Princeton

Arnett WD (1969) A Possible Model of Supernovae: Detonation of ${ }^{12} \mathrm{C}$. Astrophysics and Space Science5:180-212, DOI 10.1007/BF00650291

Arnett WD (1982) Type I supernovae. I - Analytic solutions for the early part of the light curve. ApJ253:785-797, DOI 10.1086/159681

Arnould M (1976) Possibility of synthesis of proton-rich nuclei in highly evolved stars. II. A\&A46:117-125

Audouze J, Truran JW (1975) P-process nucleosynthesis in postshock supernova envelope environments. ApJ202:204-213, DOI 10.1086/153965

Benetti S, Cappellaro E, Mazzali PA, Turatto M, Altavilla G, Bufano F, Elias-Rosa N, Kotak R, Pignata G, Salvo M, Stanishev V (2005) The Diversity of Type Ia Supernovae: Evidence for Systematics? ApJ623:1011-1016, DOI 10.1086/428608, astro-ph/0411059

Bildsten L, Hall DM (2001) Gravitational Settling of ${ }^{22} \mathrm{Ne}$ in Liquid White Dwarf Interiors. ApJ Letters549:L219-L223, DOI 10.1086/319169, astro-ph/0101365

Bodansky D, Clayton DD, Fowler WA (1968) Nuclear Quasi-Equilibrium during Silicon Burning. ApJ Supplement16:299, DOI 10.1086/190176

Böhringer H, Werner N (2010) X-ray spectroscopy of galaxy clusters: studying astrophysical processes in the largest celestial laboratories. A\&A Rev.18:127-196, DOI 10.1007/ s00159-009-0023-3

Borkowski KJ, Reynolds SP, Green DA, Hwang U, Petre R, Krishnamurthy K, Willett R (2010) Radioactive Scandium in the Youngest Galactic Supernova Remnant G1.9+0.3. ApJ Letters724:L161-L165, DOI 10.1088/2041-8205/724/2/L161, 1006.3552

Brachwitz F, Dean DJ, Hix WR, Iwamoto K, Langanke K, Martínez-Pinedo G, Nomoto K, Strayer MR, Thielemann FK, Umeda H (2000) The Role of Electron Captures in ChandrasekharMass Models for Type IA Supernovae. ApJ536:934-947, DOI 10.1086/308968, astro-ph/ 0001464

Bravo E, García-Senz D (2009) Pulsating Reverse Detonation Models of Type Ia Supernovae. I. Detonation Ignition. ApJ695:1244-1256, DOI 10.1088/0004-637X/695/2/1244, 0901.3008

Bravo E, Martínez-Pinedo G (2012) Sensitivity study of explosive nucleosynthesis in type Ia supernovae: Modification of individual thermonuclear reaction rates. Phys. Rev. C85(5):055805, DOI 10.1103/PhysRevC.85.055805, 1204.1981

Bravo E, García-Senz D, Cabezón RM, Domínguez I (2009) Pulsating Reverse Detonation Models of Type Ia Supernovae. II. Explosion. ApJ695:1257-1272, DOI 10.1088/0004-637X/695/2/ 1257, 0901.3013

Burbidge EM, Burbidge GR, Fowler WA, Hoyle F (1957) Synthesis of the Elements in Stars. Reviews of Modern Physics 29:547-650, DOI 10.1103/RevModPhys.29.547

Calder AC, Townsley DM, Seitenzahl IR, Peng F, Messer OEB, Vladimirova N, Brown EF, Truran JW, Lamb DQ (2007) Capturing the Fire: Flame Energetics and Neutronization for Type Ia Supernova Simulations. ApJ656:313-332, DOI 10.1086/510709, astro-ph/0611009 
Calder AC, Krueger BK, Jackson AP, Townsley DM (2013) The influence of chemical composition on models of Type Ia supernovae. Frontiers of Physics 8:168-188, DOI 10.1007/ s11467-013-0301-4, 1303.2207

Cameron AGW (1957) Nuclear Reactions in Stars and Nucleogenesis. PASP69:201, DOI 10.1086/ 127051

Carlton AK, Borkowski KJ, Reynolds SP, Hwang U, Petre R, Green DA, Krishnamurthy K, Willett R (2011) Expansion of the Youngest Galactic Supernova Remnant G1.9+0.3. ApJ Letters737:L22, DOI 10.1088/2041-8205/737/1/L22, 1106.4498

Chamulak DA, Brown EF, Timmes FX, Dupczak K (2008) The Reduction of the Electron Abundance during the Pre-explosion Simmering in White Dwarf Supernovae. ApJ677:160-168, DOI 10.1086/528944,0801.1643

Churazov E, Sunyaev R, Isern J, Knödlseder J, Jean P, Lebrun F, Chugai N, Grebenev S, Bravo E, Sazonov S, Renaud M (2014) Cobalt-56 $\gamma$-ray emission lines from the type Ia supernova 2014J. Nature512:406-408, DOI 10.1038/nature13672, 1405.3332

Churazov E, Sunyaev R, Isern J, Bikmaev I, Bravo E, Chugai N, Grebenev S, Jean P, Knödlseder J, Lebrun F, Kuulkers E (2015) Gamma-rays from Type Ia Supernova SN2014J. ApJ812:62, DOI 10.1088/0004-637X/812/1/62, 1502.00255

Ciaraldi-Schoolmann F, Seitenzahl IR, Röpke FK (2013) A subgrid-scale model for deflagrationto-detonation transitions in Type Ia supernova explosion simulations. Numerical implementation. A\&A559:A117, DOI 10.1051/0004-6361/201321480, 1307.8146

Crocker RM, Ruiter AJ, Seitenzahl IR, Panther FH, Baumgardt H, Moller A, Nataf DM, Ferrario L, Eldridge JJ, White M, Sim S, Tucker BE, Aharonian F (2016) Sub-luminous '1991bg-Like' Thermonuclear Supernovae Account for Most Diffuse Antimatter in the Milky Way. ArXiv e-prints 1607.03495

de Plaa J (2013) The origin of the chemical elements in cluster cores. Astronomische Nachrichten 334:416, DOI 10.1002/asna.201211870, 1210.1093

de Plaa J, Werner N, Bleeker JAM, Vink J, Kaastra JS, Méndez M (2007) Constraining supernova models using the hot gas in clusters of galaxies. A\&A465:345-355, DOI 10.1051/0004-6361: 20066382, astro-ph/0701553

Dhawan S, Leibundgut B, Spyromilio J, Blondin S (2016) A reddening-free method to estimate the ${ }^{56} \mathrm{Ni}$ mass of Type Ia supernovae. A\&A588:A84, DOI 10.1051/0004-6361/201527201, 1601. 04874

Diehl R, Siegert T, Hillebrandt W, Grebenev SA, Greiner J, Krause M, Kromer M, Maeda K, Röpke F, Taubenberger S (2014) Early ${ }^{56} \mathrm{Ni}$ decay gamma rays from SN2014J suggest an unusual explosion. Science 345:1162-1165, DOI 10.1126/science.1254738, 1407.3061

Dopita MA, Sutherland RS (2003) Astrophysics of the diffuse universe

Dupke RA, White RE III (2000) Constraints on Type IA Supernova Models from X-Ray Spectra of Galaxy Clusters. ApJ528:139-144, DOI 10.1086/308181, astro-ph/9907343

Dwek E (2016) Iron: A Key Element for Understanding the Origin and Evolution of Interstellar Dust. ArXiv e-prints 1605.01957

Fink M, Röpke FK, Hillebrandt W, Seitenzahl IR, Sim SA, Kromer M (2010) Doubledetonation sub-Chandrasekhar supernovae: can minimum helium shell masses detonate the core? A\&A514:A53, DOI 10.1051/0004-6361/200913892,1002.2173

Fink M, Kromer M, Seitenzahl IR, Ciaraldi-Schoolmann F, Röpke FK, Sim SA, Pakmor R, Ruiter AJ, Hillebrandt W (2014) Three-dimensional pure deflagration models with nucleosynthesis and synthetic observables for Type Ia supernovae. MNRAS438:1762-1783, DOI $10.1093 / \mathrm{mnras} / \mathrm{stt} 2315,1308.3257$

Foley RJ, Chornock R, Filippenko AV, Ganeshalingam M, Kirshner RP, Li W, Cenko SB, Challis PJ, Friedman AS, Modjaz M, Silverman JM, Wood-Vasey WM (2009) SN 2008ha: An Extremely Low Luminosity and Exceptionally Low Energy Supernova. The Astronomical Journal138:376-391, DOI 10.1088/0004-6256/138/2/376, 0902.2794

Fransson C, Jerkstrand A (2015) Reconciling the Infrared Catastrophe and Observations of SN 2011fe. ApJ Letters814:L2, DOI 10.1088/2041-8205/814/1/L2,1511.00245 
Fuller GM, Fowler WA, Newman MJ (1982) Stellar weak interaction rates for intermediate-mass nuclei. II - A = 21 to $\mathrm{A}=60$. ApJ252:715-740, DOI 10.1086/159597

Gasques LR, Afanasjev AV, Aguilera EF, Beard M, Chamon LC, Ring P, Wiescher M, Yakovlev DG (2005) Nuclear fusion in dense matter: Reaction rate and carbon burning. Phys. Rev. C72(2):025806, DOI 10.1103/PhysRevC.72.025806, astro-ph/0506386

Gasques LR, Brown EF, Chieffi A, Jiang CL, Limongi M, Rolfs C, Wiescher M, Yakovlev DG (2007) Implications of low-energy fusion hindrance on stellar burning and nucleosynthesis. Phys. Rev. C76(3):035802, DOI 10.1103/PhysRevC.76.035802

Graur O, Zurek D, Shara MM, Riess AG, Seitenzahl IR, Rest A (2016) Late-time Photometry of Type Ia Supernova SN 2012cg Reveals the Radioactive Decay of 57 Co. ApJ819:31, DOI 10.3847/0004-637X/819/1/31, 1505.00777

Hix WR, Meyer BS (2006) Thermonuclear kinetics in astrophysics. Nuclear Physics A 777:188207, DOI 10.1016/j.nuclphysa.2004.10.009, astro-ph/ 0509698

Howard WM, Meyer BS, Woosley SE (1991) A new site for the astrophysical gamma-process. ApJ Letters373:L5-L8, DOI 10.1086/186038

Iben I Jr (1981) On intermediate-mass single stars and accreting white dwarfs as sources of neutron-rich isotopes. ApJ243:987-993, DOI 10.1086/158663

Iben I Jr, Nomoto K, Tornambe A, Tutukov AV (1987) On interacting helium star-white dwarf pairs as supernova precursors. ApJ317:717-723, DOI 10.1086/165318

Iwamoto K, Brachwitz F, Nomoto K, Kishimoto N, Umeda H, Hix WR, Thielemann FK (1999) Nucleosynthesis in Chandrasekhar Mass Models for Type IA Supernovae and Constraints on Progenitor Systems and Burning-Front Propagation. ApJ Supplement125:439-462, DOI 10. 1086/313278, astro-ph/0002337

Jackson AP, Calder AC, Townsley DM, Chamulak DA, Brown EF, Timmes FX (2010) Evaluating Systematic Dependencies of Type Ia Supernovae: The Influence of Deflagration to Detonation Density. ApJ720:99-113, DOI 10.1088/0004-637X/720/1/99, 1007.1138

Jackson AP, Townsley DM, Calder AC (2014) Power-law Wrinkling Turbulence-Flame Interaction Model for Astrophysical Flames. ApJ784:174, DOI 10.1088/0004-637X/784/2/174, 1402 . 4527

Jones S, Roepke FK, Pakmor R, Seitenzahl IR, Ohlmann ST, Edelmann PVF (2016) Do electroncapture supernovae make neutron stars? First multidimensional hydrodynamic simulations of the oxygen deflagration. ArXiv e-prints 1602.05771

Kasliwal MM, Kulkarni SR, Gal-Yam A, Nugent PE, Sullivan M, Bildsten L, Yaron O, Perets HB, Arcavi I, Ben-Ami S, Bhalerao VB, Bloom JS, Cenko SB, Filippenko AV, Frail DA, Ganeshalingam M, Horesh A, Howell DA, Law NM, Leonard DC, Li W, Ofek EO, Polishook D, Poznanski D, Quimby RM, Silverman JM, Sternberg A, Xu D (2012) Calcium-rich Gap Transients in the Remote Outskirts of Galaxies. ApJ755:161, DOI 10.1088/0004-637X/755/2/161, 1111.6109

Kerzendorf WE, Taubenberger S, Seitenzahl IR, Ruiter AJ (2014) Very Late Photometry of SN 2011fe. ApJ Letters796:L26, DOI 10.1088/2041-8205/796/2/L26, 1406.6050

Khokhlov AM (1991) Delayed detonation model for type IA supernovae. A\&A245:114-128

Kobayashi C, Nomoto K (2009) The Role of Type Ia Supernovae in Chemical Evolution. I. Lifetime of Type Ia Supernovae and Metallicity Effect. ApJ707:1466-1484, DOI 10.1088/ 0004-637X/707/2/1466,0801.0215

Kobayashi C, Nomoto K, Hachisu I (2015) Subclasses of Type Ia Supernovae as the Origin of $[\alpha / \mathrm{Fe}]$ Ratios in Dwarf Spheroidal Galaxies. ApJ Letters804:L24, DOI 10.1088/2041-8205/ 804/1/L24, 1503.06739

Kromer M, Sim SA, Fink M, Röpke FK, Seitenzahl IR, Hillebrandt W (2010) Double-detonation Sub-Chandrasekhar Supernovae: Synthetic Observables for Minimum Helium Shell Mass Models. ApJ719:1067-1082, DOI 10.1088/0004-637X/719/2/1067, 1006.4489

Kromer M, Fink M, Stanishev V, Taubenberger S, Ciaraldi-Schoolman F, Pakmor R, Röpke FK, Ruiter AJ, Seitenzahl IR, Sim SA, Blanc G, Elias-Rosa N, Hillebrandt W (2013) 3D deflagration simulations leaving bound remnants: a model for 2002cx-like Type Ia supernovae. MNRAS429:2287-2297, DOI 10.1093/mnras/sts498, 1210.5243 
Kromer M, Ohlmann ST, Pakmor R, Ruiter AJ, Hillebrandt W, Marquardt KS, Röpke FK, Seitenzahl IR, Sim SA, Taubenberger S (2015) Deflagrations in hybrid CONe white dwarfs: a route to explain the faint Type Iax supernova 2008ha. MNRAS450:3045-3053, DOI 10.1093/mnras/ stv886, 1503.04292

Lambert DL (1992) The p-nuclei - Abundances and origins. A\&A Rev.3:201-256, DOI 10.1007/ BF00872527

Langanke K, Martínez-Pinedo G (2001) Rate Tables for the Weak Processes of pf-SHELL Nuclei in Stellar Environments. Atomic Data and Nuclear Data Tables 79:1-46, DOI 10.1006/adnd. 2001.0865

Livne E (1990) Successive detonations in accreting white dwarfs as an alternative mechanism for type I supernovae. ApJ Letters354:L53-L55, DOI 10.1086/185721

Livne E, Arnett D (1995) Explosions of Sub-Chandrasekhar Mass White Dwarfs in Two Dimensions. ApJ452:62, DOI 10.1086/176279

Lopez LA, Grefenstette BW, Reynolds SP, An H, Boggs SE, Christensen FE, Craig WW, Eriksen KA, Fryer CL, Hailey CJ, Harrison FA, Madsen KK, Stern DK, Zhang WW, Zoglauer A (2015) A Spatially Resolved Study of the Synchrotron Emission and Titanium in Tycho's Supernova Remnant Using NuSTAR. ApJ814:132, DOI 10.1088/0004-637X/814/2/132, 1504.07238

Maeda K, Röpke FK, Fink M, Hillebrandt W, Travaglio C, Thielemann FK (2010) Nucleosynthesis in Two-Dimensional Delayed Detonation Models of Type Ia Supernova Explosions. ApJ712:624-638, DOI 10.1088/0004-637X/712/1/624, 1002.2153

Marquardt KS, Sim SA, Ruiter AJ, Seitenzahl IR, Ohlmann ST, Kromer M, Pakmor R, Röpke FK (2015) Type Ia supernovae from exploding oxygen-neon white dwarfs. A\&A580:A118, DOI 10.1051/0004-6361/201525761, 1506.05809

Martínez-Rodríguez H, Piro AL, Schwab J, Badenes C (2016) Neutronization During Carbon Simmering In Type Ia Supernova Progenitors. ArXiv e-prints 1602.00673

Mazzali PA, Röpke FK, Benetti S, Hillebrandt W (2007) A Common Explosion Mechanism for Type Ia Supernovae. Science 315:825, DOI 10.1126/science.1136259, astro-ph/0702351

Mazzali PA, Sullivan M, Filippenko AV, Garnavich PM, Clubb KI, Maguire K, Pan YC, Shappee B, Silverman JM, Benetti S, Hachinger S, Nomoto K, Pian E (2015) Nebular spectra and abundance tomography of the Type Ia supernova SN $2011 \mathrm{fe}$ : a normal SN Ia with a stable Fe core. MNRAS450:2631-2643, DOI 10.1093/mnras/stv761,1504.04857

McWilliam A (1997) Abundance Ratios and Galactic Chemical Evolution. Annu. Rev. Astron. Astrophys35:503-556, DOI 10.1146/annurev.astro.35.1.503

Meakin CA, Seitenzahl I, Townsley D, Jordan GC IV, Truran J, Lamb D (2009) Study of the Detonation Phase in the Gravitationally Confined Detonation Model of Type Ia Supernovae. ApJ693:1188-1208, DOI 10.1088/0004-637X/693/2/1188,0806.4972

Mernier F, de Plaa J, Pinto C, Kaastra JS, Kosec P, Zhang YY, Mao J, Werner N (2016a) Origin of central abundances in the hot intra-cluster medium. i. individual and average abundance ratios from xmm-newton epic. A\&A592:A157, DOI 10.1051/0004-6361/201527824, 1606.01165

Mernier F, de Plaa J, Pinto C, Kaastra JS, Kosec P, Zhang YY, Mao J, Werner N, Pols OR, Vink J (2016b) Origin of central abundances in the hot intra-cluster medium. ii. chemical enrichment and supernova yield models. A\&A595:A126, DOI 10.1051/0004-6361/201628765, 1608.03888

Meyer BS, Krishnan TD, Clayton DD (1996) ${ }^{48} \mathrm{Ca}$ Production in Matter Expanding from High Temperature and Density. ApJ462:825, DOI 10.1086/177197

Miles BJ, van Rossum DR, Townsley DM, Timmes FX, Jackson AP, Calder AC, Brown EF (2016) On Measuring the Metallicity of a Type Ia Supernova Progenitor. ApJ824:59, DOI 10.3847/ 0004-637X/824/1/59, 1508.05961

Moll R, Raskin C, Kasen D, Woosley SE (2014) Type Ia Supernovae from Merging White Dwarfs. I. Prompt Detonations. ApJ785:105, DOI 10.1088/0004-637X/785/2/105, 1311.5008

Moore K, Townsley DM, Bildsten L (2013) The Effects of Curvature and Expansion on Helium Detonations on White Dwarf Surfaces. ApJ776:97, DOI 10.1088/0004-637X/776/2/97, 1308 . 4193 
Nomoto K, Thielemann FK, Yokoi K (1984) Accreting white dwarf models of Type I supernovae. III - Carbon deflagration supernovae. ApJ286:644-658, DOI 10.1086/162639

Ohlmann ST, Kromer M, Fink M, Pakmor R, Seitenzahl IR, Sim SA, Röpke FK (2014) The white dwarf's carbon fraction as a secondary parameter of Type Ia supernovae. A\&A572:A57, DOI 10.1051/0004-6361/201423924, 1409.2866

Pakmor R, Kromer M, Röpke FK, Sim SA, Ruiter AJ, Hillebrandt W (2010) Sub-luminous type Ia supernovae from the mergers of equal-mass white dwarfs with mass $\sim 0.9 \mathrm{M}_{\text {solar }}$. Nature463:6164, DOI 10.1038/nature08642,0911.0926

Pakmor R, Kromer M, Taubenberger S, Sim SA, Röpke FK, Hillebrandt W (2012) Normal Type Ia Supernovae from Violent Mergers of White Dwarf Binaries. ApJ Letters747:L10, DOI 10. 1088/2041-8205/747/1/L10, 1201.5123

Pakmor R, Kromer M, Taubenberger S, Springel V (2013) Helium-ignited Violent Mergers as a Unified Model for Normal and Rapidly Declining Type Ia Supernovae. ApJ Letters770:L8, DOI 10.1088/2041-8205/770/1/L8, 1302.2913

Papish O, Perets HB (2016) Supernovae from Direct Collisions of White Dwarfs and the Role of Helium Shell Ignition. ApJ822:19, DOI 10.3847/0004-637X/822/1/19, 1502.03453

Parikh A, José J, Seitenzahl IR, Röpke FK (2013) The effects of variations in nuclear interactions on nucleosynthesis in thermonuclear supernovae. A\&A557:A3, DOI 10.1051/0004-6361/ 201321518, 1306.6007

Pignatari M, Göbel K, Reifarth R, Travaglio C (2016) The production of proton-rich isotopes beyond iron: The $\gamma$-process in stars. International Journal of Modern Physics E 25:1630003232, DOI 10.1142/S0218301316300034, 1605.03690

Pinto PA, Eastman RG (2000) The Physics of Type IA Supernova Light Curves. I. Analytic Results and Time Dependence. ApJ530:744-756, DOI 10.1086/308376

Piro AL, Bildsten L (2008) Neutronization during Type Ia Supernova Simmering. ApJ673:1009_ 1013 , DOI $10.1086 / 524189,0710.1600$

Plewa T, Calder AC, Lamb DQ (2004) Type Ia Supernova Explosion: Gravitationally Confined Detonation. ApJ Letters612:L37-L40, DOI 10.1086/424036, astro-ph/ 0405163

Rauscher T, Dauphas N, Dillmann I, Fröhlich C, Fülöp Z, Gyürky G (2013) Constraining the astrophysical origin of the p-nuclei through nuclear physics and meteoritic data. Reports on Progress in Physics 76(6):066201, DOI 10.1088/0034-4885/76/6/066201, 1303.2666

Röpke FK, Hillebrandt W, Schmidt W, Niemeyer JC, Blinnikov SI, Mazzali PA (2007) A Three-Dimensional Deflagration Model for Type Ia Supernovae Compared with Observations. ApJ668:1132-1139, DOI 10.1086/521347,0 0707.1024

Röpke FK, Kromer M, Seitenzahl IR, Pakmor R, Sim SA, Taubenberger S, Ciaraldi-Schoolmann F, Hillebrandt W, Aldering G, Antilogus P, Baltay C, Benitez-Herrera S, Bongard S, Buton C, Canto A, Cellier-Holzem F, Childress M, Chotard N, Copin Y, Fakhouri HK, Fink M, Fouchez D, Gangler E, Guy J, Hachinger S, Hsiao EY, Chen J, Kerschhaggl M, Kowalski M, Nugent P, Paech K, Pain R, Pecontal E, Pereira R, Perlmutter S, Rabinowitz D, Rigault M, Runge K, Saunders C, Smadja G, Suzuki N, Tao C, Thomas RC, Tilquin A, Wu C (2012) Constraining Type Ia Supernova Models: SN 2011fe as a Test Case. ApJ Letters750:L19, DOI 10.1088/ 2041-8205/750/1/L19, 1203.4839

Ruiter AJ, Sim SA, Pakmor R, Kromer M, Seitenzahl IR, Belczynski K, Fink M, Herzog M, Hillebrandt W, Röpke FK, Taubenberger S (2013) On the brightness distribution of Type Ia supernovae from violent white dwarf mergers. MNRAS429:1425-1436, DOI 10.1093/mnras/sts423, 1209.0645

Scalzo RA, Aldering G, Antilogus P, Aragon C, Bailey S, Baltay C, Bongard S, Buton C, Childress M, Chotard N, Copin Y, Fakhouri HK, Gal-Yam A, Gangler E, Hoyer S, Kasliwal M, Loken S, Nugent P, Pain R, Pécontal E, Pereira R, Perlmutter S, Rabinowitz D, Rau A, Rigaudier G, Runge K, Smadja G, Tao C, Thomas RC, Weaver B, Wu C (2010) Nearby Supernova Factory Observations of SN 2007if: First Total Mass Measurement of a Super-Chandrasekhar-Mass Progenitor. ApJ713:1073-1094, DOI 10.1088/0004-637X/713/2/1073, 1003.2217 
Scalzo RA, Ruiter AJ, Sim SA (2014) The ejected mass distribution of Type Ia supernovae: a significant rate of non-Chandrasekhar-mass progenitors. MNRAS445:2535-2544, DOI 10.1093/mnras/stu1808, 1408.6601

Schmidt W, Niemeyer JC, Hillebrandt W, Röpke FK (2006) A localised subgrid scale model for fluid dynamical simulations in astrophysics. II. Application to type Ia supernovae. A\&A450:283-294, DOI 10.1051/0004-6361:20053618, astro-ph/0601500

Seitenzahl IR, Meakin CA, Townsley DM, Lamb DQ, Truran JW (2009a) Spontaneous Initiation of Detonations in White Dwarf Environments: Determination of Critical Sizes. ApJ696:515-527, DOI 10.1088/0004-637X/696/1/515,0901.3677

Seitenzahl IR, Taubenberger S, Sim SA (2009b) Late-time supernova light curves: the effect of internal conversion and Auger electrons. MNRAS400:531-535, DOI 10.1111/j.1365-2966.2009. 15478.x, 0908.0247

Seitenzahl IR, Townsley DM, Peng F, Truran JW (2009c) Nuclear statistical equilibrium for Type Ia supernova simulations. Atomic Data and Nuclear Data Tables 95:96-114, DOI 10.1016/j. adt.2008.08.001

Seitenzahl IR, Röpke FK, Fink M, Pakmor R (2010) Nucleosynthesis in thermonuclear supernovae with tracers: convergence and variable mass particles. MNRAS407:2297-2304, DOI 10.1111/ j.1365-2966.2010.17106.x, 1005.5071

Seitenzahl IR, Ciaraldi-Schoolmann F, Röpke FK (2011) Type Ia supernova diversity: white dwarf central density as a secondary parameter in three-dimensional delayed detonation models. MNRAS414:2709-2715, DOI 10.1111/j.1365-2966.2011.18588.x, 1012.4929

Seitenzahl IR, Cescutti G, Röpke FK, Ruiter AJ, Pakmor R (2013a) Solar abundance of manganese: a case for near Chandrasekhar-mass Type Ia supernova progenitors. A\&A559:L5, DOI 10.1051/ 0004-6361/201322599, 1309.2397

Seitenzahl IR, Ciaraldi-Schoolmann F, Röpke FK, Fink M, Hillebrandt W, Kromer M, Pakmor R, Ruiter AJ, Sim SA, Taubenberger S (2013b) Three-dimensional delayed-detonation models with nucleosynthesis for Type Ia supernovae. MNRAS429:1156-1172, DOI 10.1093/mnras/ sts402, 1211.3015

Seitenzahl IR, Summa A, Krauß F, Sim SA, Diehl R, Elsässer D, Fink M, Hillebrandt W, Kromer M, Maeda K, Mannheim K, Pakmor R, Röpke FK, Ruiter AJ, Wilms J (2015) 5.9-keV Mn Kshell X-ray luminosity from the decay of ${ }^{55} \mathrm{Fe}$ in Type Ia supernova models. MNRAS447:14841490, DOI 10.1093/mnras/stu2537, 1412.0835

Seitenzahl IR, Kromer M, Ohlmann ST, Ciaraldi-Schoolmann F, Marquardt K, Fink M, Hillebrandt W, Pakmor R, Roepke FK, Ruiter AJ, Sim SA, Taubenberger S (2016) Three-dimensional simulations of gravitationally confined detonations compared to observations of SN 1991T. ArXiv e-prints 1606.00089

Shen KJ, Bildsten L (2014) The Ignition of Carbon Detonations via Converging Shock Waves in White Dwarfs. ApJ785:61, DOI 10.1088/0004-637X/785/1/61, 1305.6925

Shen KJ, Moore K (2014) The Initiation and Propagation of Helium Detonations in White Dwarf Envelopes. ApJ797:46, DOI 10.1088/0004-637X/797/1/46, 1409.3568

Sim SA, Röpke FK, Hillebrandt W, Kromer M, Pakmor R, Fink M, Ruiter AJ, Seitenzahl IR (2010) Detonations in Sub-Chandrasekhar-mass C+O White Dwarfs. ApJ Letters714:L52-L57, DOI 10.1088/2041-8205/714/1/L52, 1003.2917

Stein J, Wheeler JC (2006) The Convective Urca Process with Implicit Two-dimensional Hydrodynamics. ApJ643:1190-1197, DOI 10.1086/503246, astro-ph/0512580

Stritzinger M, Mazzali PA, Sollerman J, Benetti S (2006) Consistent estimates of ${ }^{56} \mathrm{Ni}$ yields for type Ia supernovae. A\&A460:793-798, DOI 10.1051/0004-6361:20065514, astro-ph/ 0609232

Taam RE (1980) The long-term evolution of accreting carbon white dwarfs. ApJ242:749-755, DOI 10.1086/158509

Taubenberger S, Elias-Rosa N, Kerzendorf WE, Hachinger S, Spyromilio J, Fransson C, Kromer M, Ruiter AJ, Seitenzahl IR, Benetti S, Cappellaro E, Pastorello A, Turatto M, Marchetti A (2015) Spectroscopy of the Type Ia supernova 2011fe past $1000 \mathrm{~d}$. MNRAS448:L48-L52, DOI 10.1093/mnrasl/slu201, 1411.7599 
Thielemann FK, Nomoto K, Yokoi K (1986) Explosive nucleosynthesis in carbon deflagration models of Type I supernovae. A\&A158:17-33

Timmes FX, Woosley SE, Weaver TA (1995) Galactic chemical evolution: Hydrogen through zinc. ApJ Supplement98:617-658, DOI 10.1086/192172, astro-ph/9411003

Timmes FX, Brown EF, Truran JW (2003) On Variations in the Peak Luminosity of Type Ia Supernovae. ApJ Letters590:L83-L86, DOI 10.1086/376721, astro-ph/0305114

Townsley DM, Miles BJ, Timmes FX, Calder AC, Brown EF (2016) A Tracer Method for Computing Type Ia Supernova Yields: Burning Model Calibration, Reconstruction of Thickened Flames, and Verification for Planar Detonations. ApJ Supplement225:3, DOI 10.3847/ 0067-0049/225/1/3, 1605.04878

Travaglio C, Hillebrandt W, Reinecke M, Thielemann FK (2004) Nucleosynthesis in multidimensional SN Ia explosions. A\&A425:1029-1040, DOI 10.1051/0004-6361:20041108, astro-ph/0406281

Travaglio C, Röpke FK, Gallino R, Hillebrandt W (2011) Type Ia Supernovae as Sites of the p-process: Two-dimensional Models Coupled to Nucleosynthesis. ApJ739:93, DOI 10.1088/ 0004-637X/739/2/93, 1106.0582

Travaglio C, Gallino R, Rauscher T, Röpke FK, Hillebrandt W (2015) Testing the Role of SNe Ia for Galactic Chemical Evolution of p-nuclei with Two-dimensional Models and with s-process Seeds at Different Metallicities. ApJ799:54, DOI 10.1088/0004-637X/799/1/54, 1411.2399

Troja E, Segreto A, La Parola V, Hartmann D, Baumgartner W, Markwardt C, Barthelmy S, Cusumano G, Gehrels N (2014) Swift/BAT Detection of Hard X-Rays from Tycho's Supernova Remnant: Evidence for Titanium-44. ApJ Letters797:L6, DOI 10.1088/2041-8205/797/1/L6, 1411.0991

Tutukov A, Yungelson L (1996) Double-degenerate semidetached binaries with helium secondaries: cataclysmic variables, supersoft X-ray sources, supernovae and accretion-induced collapses. MNRAS280:1035-1045, DOI 10.1093/mnras/280.4.1035

Waldman R, Sauer D, Livne E, Perets H, Glasner A, Mazzali P, Truran JW, Gal-Yam A (2011) Helium Shell Detonations on Low-mass White Dwarfs as a Possible Explanation for SN 2005E. ApJ738:21, DOI 10.1088/0004-637X/738/1/21, 1009.3829

Woosley SE (1997) Neutron-rich Nucleosynthesis in Carbon Deflagration Supernovae. ApJ476:801-810

Woosley SE, Howard WM (1978) The p-process in supernovae. ApJ Supplement36:285-304, DOI $10.1086 / 190501$

Woosley SE, Kasen D (2011) Sub-Chandrasekhar Mass Models for Supernovae. ApJ734:38, DOI 10.1088/0004-637X/734/1/38, 1010.5292

Woosley SE, Weaver TA (1994) Sub-Chandrasekhar mass models for Type IA supernovae. ApJ423:371-379, DOI 10.1086/173813

Woosley SE, Arnett WD, Clayton DD (1973) The Explosive Burning of Oxygen and Silicon. ApJ Supplement26:231, DOI 10.1086/190282

Yamaguchi H, Badenes C, Foster AR, Bravo E, Williams BJ, Maeda K, Nobukawa M, Eriksen KA, Brickhouse NS, Petre R, Koyama K (2015) A Chandrasekhar Mass Progenitor for the Type Ia Supernova Remnant 3C 397 from the Enhanced Abundances of Nickel and Manganese. ApJ Letters801:L31, DOI 10.1088/2041-8205/801/2/L31, 1502.04255 